\title{
A Plan Recognizing Algorithm based on Fuzzy Cognitive Plan Map
}

\author{
Yuan Feng ${ }^{\mathrm{a}}$, Zengyu Cai ${ }^{\mathrm{a}}$, Xuhui Wang ${ }^{\mathrm{a}}$, Jianwei Zhang ${ }^{\mathrm{a}, \mathrm{b}, *}$, Yong Gan ${ }^{\mathrm{a}}$ \\ ${ }^{a}$ School of Computer and Communication Engineering, Zhengzhou University of Light Industry, Zhengzhou, 450002, China \\ ${ }^{b}$ School of Software Engineering, Zhengzhou University of Light Industry, Zhengzhou, 450002, China
}

\begin{abstract}
With plan recognition applications continuously expanding, the speed of traditional plan recognition is too slow, which hampers plan recognition applying to some areas. So, it is important to construct simple and efficient plan recognizing algorithm. Based on the study of plan knowledge graph, the concept of fuzzy cognitive plan map is proposed. Then, the recognizing algorithm based on fuzzy cognitive plan map is proposed. In this algorithm, it uses the operation of matrix to recognize agent's plan, which can overcome the inefficiency of searching graph in plan knowledge graph. Experimental results show that the plan recognizing algorithm has excellent performance in plan recognition and the same recognition result is true for plan knowledge graph and it can reduce the recognition time greatly. The algorithm of plan recognition does well in intelligent game, smart home, network security and other fields, which has an important significance for extending the application area of plan recognition.
\end{abstract}

Keywords: intelligent plan; plan recognizing; cognitive plan map; fuzzy

(Submitted on July 25, 2017; Revised on August 30, 2017; Accepted on September 15, 2017)

(This paper was presented at the Third International Symposium on System and Software Reliability.)

(C) 2017 Totem Publisher, Inc. All rights reserved.

\section{Introduction}

Planning recognition is one of the earlier research areas in artificial intelligence. It is an important branch of the field of artificial intelligence and has a wide range of applications. Early planning recognition methods include story understanding [8], psychology [15], natural language recognition [13], conversation analysis [12], and so on. Plan recognition has become a focus of artificial intelligence research. There are many achievements of plan recognition [3,4,5,6,7,11].

Planning recognition inference agent's target based on the observed action, but sometimes it is difficult to infer the target of the agent according to part of the observation action without two meanings. In order to overcome this difficulty of planning recognition, many scholars have studied it deeply, and proposed a variety of planning recognition methods. The typical planning recognition is Kautz's plan recognition universal framework [9]. Charniak [7] applied the Bayesian network to planning recognition, and proposed a planning recognition model based on Bayesian networks. It makes the method of planning recognition become more widely used. Jiang proposed a recognition method based on planning knowledge graphs [11]. This method can transform the planning problem into a graph search problem, and is more effective than the Kautz's method. Owing to the advantage of plan knowledge graph, there are many achievements based on it, such as misleading action processing [3], predicting future actions [5], and intrusion intention recognition [6]. But the PKG is still complicated. Searching goals form graph is a time-consuming task, especially when there are massive nodes in PKG.

The Cognitive Map (CM) has its advantages, such as interpreting well, representing intuitively and reasoning fast. It is widely used in many fields [1,2,10]. By taking advantage of CM 's reasoning fast, it proposes the concept of Fuzzy Cognitive Plan Map (FCPM) and the recognizing algorithm is based on FCPM. The recognizing algorithm based on FCPM uses the operation of matrix to recognize agent's plan, which can overcome the inefficiency of searching graph in plan knowledge graph. It has an important significance for extending the application area of plan recognition.

* Corresponding author.

E-mail address: mailczy@163.com. 


\section{Preliminaries}

\subsection{Plan and Plan Recognition}

Planning recognition is a process by observing the activity of agent to infer their activities [11]. The essence of it is to reason out a reasonable, complete and comprehensive planning by observing the fragments and trivial phenomena. In Intrusion detection, according to the user's action or network packets, we determine whether the intrusion occurred or not, and predict the next target of the intruder.

Essentially, planning is an action sequence of agent [14]. A planning problem involves the following four sets: the Operators set, the Objects set, the Initial Conditions set and the Goals set, where each element of the Initial Conditions set and the Goals set is a proposition. The function of intelligent planning is to implement what kind of action on the object under the initial conditions, so that the target of the problem can be achieved, and the sequence of found actions is called the planning solution.

\subsection{Plan Knowledge Graph and Supporting Degree}

Plan knowledge graph is a non-loop and/or graph, where nodes represent planning (events) [11]. The AND node indicates that the child node is part of the parent node and that there is a whole-Part relationship between the child node and its parent, and it is marked with arcs in the graph. OR nodes represent an abstract and specific relationship between their parent nodes and their child nodes. This kind of planning recognition algorithm, based on the planning knowledge graph, transforms the planning recognition problem into the graph search problem. Therefore, the recognition efficiency is higher, but it's more complicated. References [11] provide the support degree of the two relationships, the support degree of specific node for the abstract node is 1 , and the sum support degree of part of the planning for the overall planning is 1 , cooking PKG as shown in Figure 1.

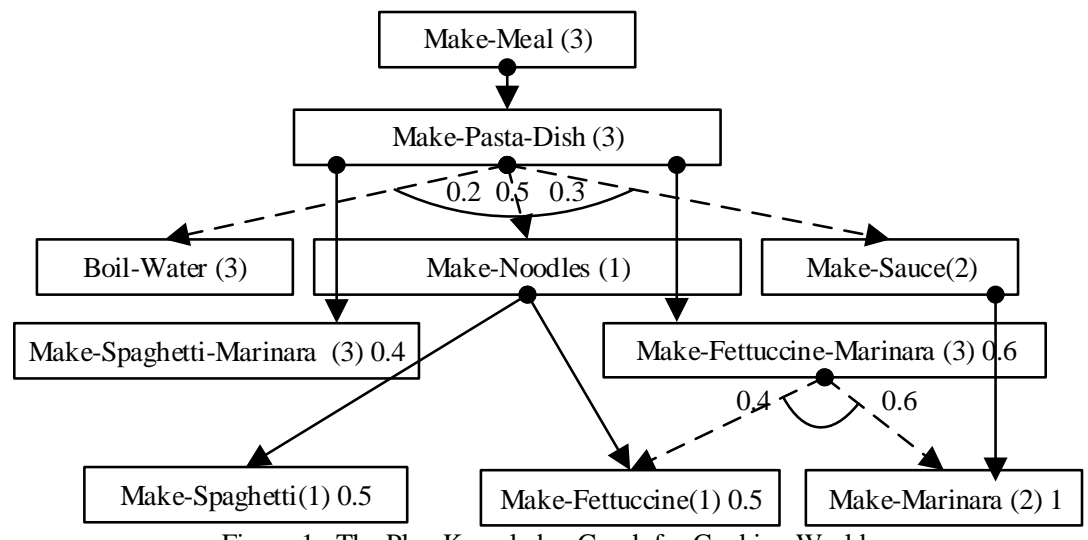

Figure 1. The Plan Knowledge Graph for Cooking World

\subsection{Cognitive Map and Fuzzy Cognitive Map}

In recent years, Cognitive Map, also called Mental Map (MM), is one of the research hot pots. The Cognitive Map begins with cognitive psychology. It is people's inner expression of the real world and it is a large amount of knowledge of the spatial structure of the environment in one's mind. Cognitive Map has been studied in various fields [1,2,10], such as psychology, education, archaeology, planning, geography, urban planning, management and history, etc. Cognitive Map is a graphical model for expressing and reasoning the causal relationship between concepts in system, and the nodes and edges represent the causal relationship between concepts and concepts respectively. Because Fuzzy Cognitive Map (FCM) is used to express and reason Fuzzy Causal Relation between concepts, the expression and reasoning ability of FCM is stronger, and it is the mainstream of Cognitive Map research at present.

\section{Plan Recognizing Algorithm Based On Fuzzy Cognitive Plan Map}

\subsection{Basic Concept}

Cognitive Map (CM): The Cognitive map is consisted by a node set $\mathrm{C}$ and an arrow set $A$, as $C M=(C, A)$. A node expresses a concept, which is the goal or function of the system. And the arrow expresses the causal relationship between the concepts. 
Node of Cognitive Map: It's also called concept node. The weights between concept nodes express the causal relationship between the concepts.

Fuzzy Cognitive Map (FCM): FCM is a fuzzy directed graph. It is constituted by nodes and edges. It is denoted by $F C M=(V, E, W)$. The $V=\left\{v_{1}, v_{2}, \ldots, v_{n}\right\}$, is the concept node set. The $E=\left\{\left\langle v_{i}, v_{j}\right\rangle\right\} v_{i}, v_{j} \in V$, is the directed arc set. The $\left\langle v_{i}\right.$, $v_{j}>$ is the causal correlation of $v_{i}$ to $v_{j}$. The $W=\left\{w_{i j} \mid w_{i j}\right.$ is the weight of $\left.\left\langle v_{i}, v_{j}\right\rangle\right\}$, The $w_{i j}$ is the supporting degree of $v_{i}$ to $v_{j}$. The adjacency matrix of $F C M$ is denoted as EFCM. So the $F C M$ also can be denoted as $(V, E)$.

Simple Fuzzy Cognitive Map (SFCM): If the FCM's edge weight is range $\{-1,0,1\}$, it is called SFCM.

Fuzzy Cognitive Plan Map (FCPM): The FCPM consists with tow cognitive map. The FCPM is denoted by C (FCM, $S F C M$ ). Both the FCM and SFCM are acyclic graph. The $F C M$ expresses part-whole relation, with range $\{-1,0,1\}$. And the $S F C M$ expresses abstract-special relation, with range $[-1,1]$.

Plan Recognition Problem: a Plan Recognition Problem based on Plan Cognitive Map is described by a triple $<V$, $C$, $O>$. The $V$ is the concept node set, in which there are all the plan or event in domain. The $C$ is the Plan Cognitive Map (FCM , SFCM). The $O$ is the instantaneous state vector of $V$. It expresses the observed events, in which the values corresponding to observed events are equal 1 .

Reasoning Process: For a plan problem $\langle V, C, O\rangle$, make the $\mathrm{A}_{\mathrm{i}}$ be the instantaneous state vector, $\mathrm{C}$ as $\langle F C M, S F C M\rangle$, the $\mathrm{E}_{\mathrm{FCM}}$ and $\mathrm{E}_{\mathrm{SFCM}}$ is respectively the Incidence Matrix of FCM and SFCM. After once reasoning, the instantaneous state vector is $A_{i+l}$. The $A_{i+1}$ is calculate as (1):

$$
A_{i+l}=\left(A_{i} \times E_{F C M}\right) U\left(A_{i} \bullet E_{S F C M}\right) U A_{i}
$$

\subsection{Construction for FCPM}

The FCPM can be directly constructed according to the field knowledge. It is similar to the construction of PKG. First, abstract the entire plan in one field. Secondly, analyze the abstraction-specialization and whole-part relationships between each plan. And then, determine correlation strengths between plans. Finally, construct the complete FCPM according to the relationships and correlation strengths. The PKG is a classical knowledge representation method of planning. It is widely used in many fields and its structure is similar to FCPM's. As there have been lots of PFGs, it proposes the conversion algorithm between PKG and FCPM to use them conveniently. The algorithm is shown in Figure 2.

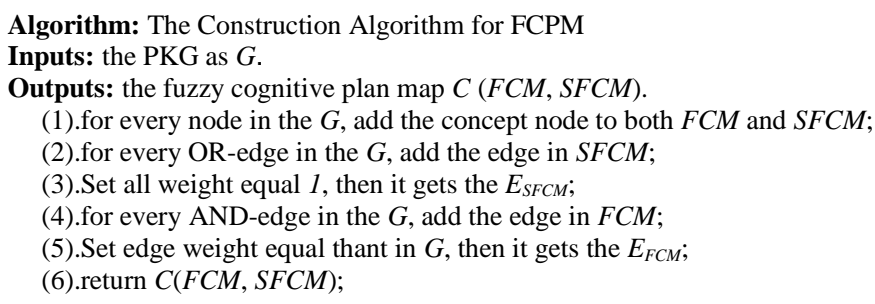

Figure 2. The Construction Algorithm for FCPM

\subsection{Plan Recognizing Algorithm based on Fuzzy Cognitive Plan Map}

The function of plan recognizing algorithm based on FCPM is recognizing the agent's goal according to the fuzzy cognitive plan map, observed events and their reliability. First, it initializes the instantaneous state vector of concept nodes as $A$. And then, it does the loop that $A$ equals to the union operation of itself, $A$ left multiplication ESFCM and A composition EFCM, until $A$ remains unchanged forever. The it gets the state vector $A$ ', it contains the all probability of the plans. The plan recognizing algorithm based on FCPM is shown in Figure 3.

\section{Experimental Results And Discussion}

\subsection{Time Complexity}

To evaluate the effectiveness of our algorithms and compare it with PKG, we applied them to JAVA and got the tested results on a PC with a Intel i3-4170 processor, 4GB memory and Windows 7 Home Premium. The running time of our algorithms is too short to get the absolute value. It tacks theft for example. It used the average value of running 10000 times 
as running time. To clarify the influence of intrusion action to the time, we gradually increased the knowledge quantity. The CPU second per recognition is the average time it takes to process a set of observed actions.

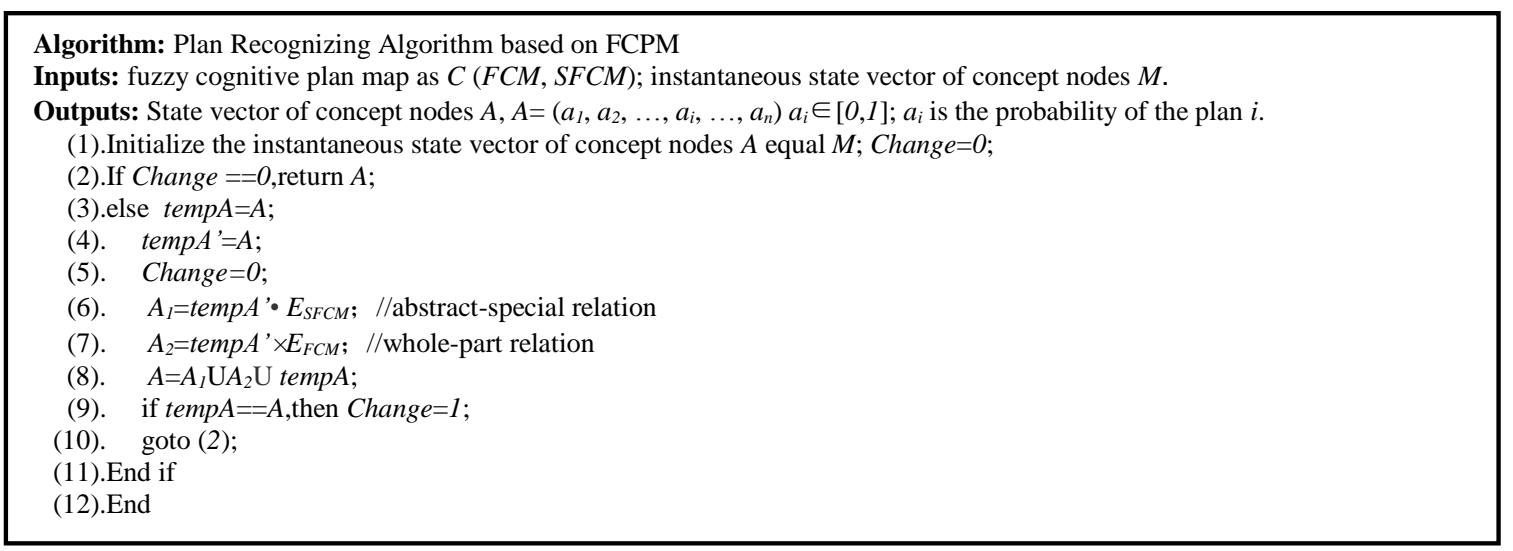

Figure 3. The Plan Recognizing Algorithm based on FCPM

The empirical results are shown in Figure 4. For PKG and FCPM, there were both linear relationship between CPU nanosecond per update and number of nodes. That is, the recognition time and node number are linear relations both in PKG and FCPM. The running time of PKG is more than our method's. So the FCPM is better than PKG in recognition speed. The plan recognition based on PKG is a time-consuming work because the inefficiency in searching graph. The FCPM can recognize plan efficiently by the operations of matrix product and matrix combination. So plan recognition based on FCPM has favorable expansibility and can be used in more fields.

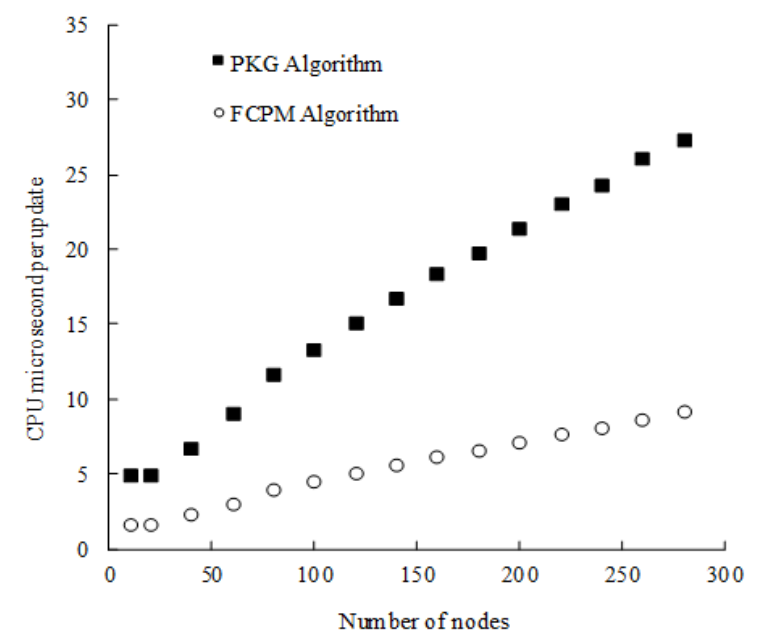

Figure 4. Experimental Results of Time Complexity Evaluation Case Analysis for Cooking Domain

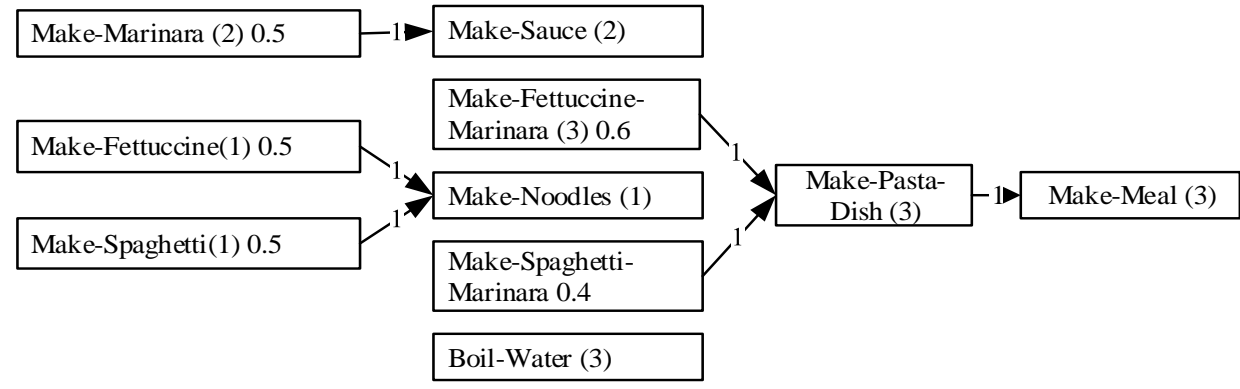

Figure 5. The SFCM For Cooking Domain 
The cooking domain is a classical field of plan recognition. For the sake of comparing with the methods based on PKG, its knowledge expression is the same to Referenc [11]. The PKG is shown in Figure 1. The FCPM can be constructed by Algorithm 1. The FCPM is composed by a SFCM and a FCM. They are shown in Figure 5 and Figure 6 respectively.

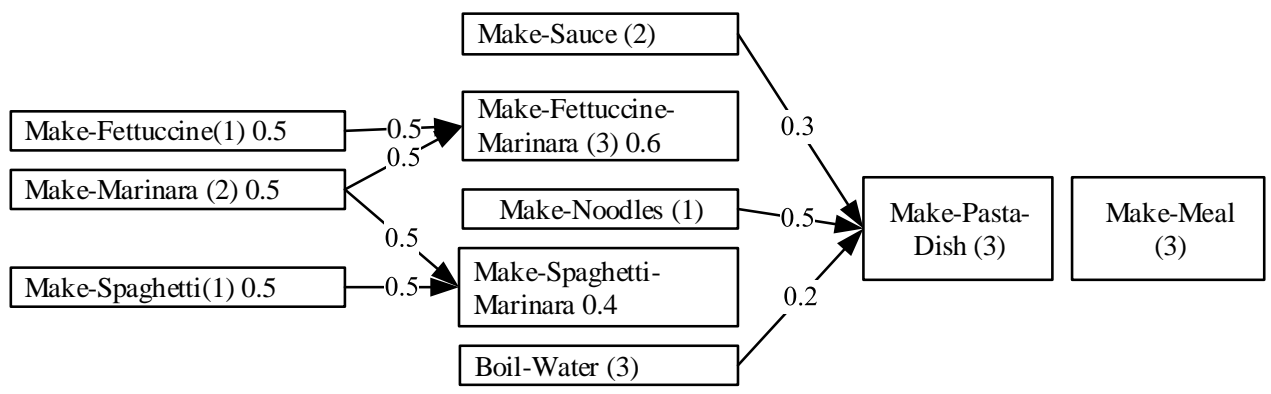

Figure 6. The FCM for Cooking Domain

Figure 5 is the SFCM of FCPM of Cooking world. It corresponds to the whole-parts relationship in PKG. Its incidence matrix is as follows:

$$
S F C M=\left|\begin{array}{llllllllll}
0 & 0 & 0 & 0 & 0 & 0 & 0 & 0 & 0 & 0 \\
1 & 0 & 0 & 0 & 0 & 0 & 0 & 0 & 0 & 0 \\
0 & 0 & 0 & 0 & 0 & 0 & 0 & 0 & 0 & 0 \\
0 & 1 & 0 & 0 & 0 & 0 & 0 & 0 & 0 & 0 \\
0 & 0 & 0 & 0 & 0 & 0 & 0 & 0 & 0 & 0 \\
0 & 1 & 0 & 0 & 0 & 0 & 0 & 0 & 0 & 0 \\
0 & 0 & 0 & 0 & 0 & 0 & 0 & 0 & 0 & 0 \\
0 & 0 & 1 & 0 & 0 & 0 & 0 & 0 & 0 & 0 \\
0 & 0 & 0 & 0 & 1 & 0 & 0 & 0 & 0 & 0 \\
0 & 0 & 0 & 0 & 1 & 0 & 0 & 0 & 0 & 0
\end{array}\right|
$$

Figure 6 is the FCM of FCPM of Cooking world. It corresponds to the abstraction-specialization relationship in PKG. Its incidence matrix is as follows:

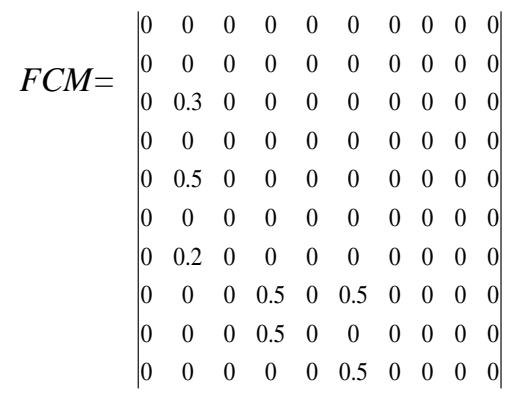

The recognized results by the plan recognizing algorithm based on FCPM is shown in Table 1. Compared with Reference[11], our algorithm is by the premise of improving recognition speed. It is in full accord with Reference[11]. So, it is superior to the algorithm in Reference [11].

Table 1. Recognized Results by Algorithm Based on FCPM

\begin{tabular}{|c|c|}
\hline Events & $\begin{array}{c}\text { probability of the } \\
\text { events }\end{array}$ \\
\hline Make-Meal & 0.8 \\
\hline Make-Pasta-Dish & 0.8 \\
\hline Boil-Water & 0 \\
\hline Make-Noodles & 1 \\
\hline Make-Sauce & 1 \\
\hline Make-Fettucine-Marinara & 1 \\
\hline Make-Fettucine & 1 \\
\hline Make-Marinara & 1 \\
\hline
\end{tabular}




\subsection{Comparison With Existing Algorithms}

The Kautz method is one of the most classical methods of planning recognition. The PKG method is an improvement to the Kautz's, which overcomes the defect of the Kautz's method, simplifies the identification method and introduces the Bayesian theory. Plus, it can handle probabilistic knowledge. The PFA method improved the PKG method and increased the ability to predict future actions, thereby the PKG method is improved in function. In this paper, the FCPM method introduces fuzzy cognitive map into the field of planning recognition, and the planning recognition process is realized by the operation of the matrix, and this FCPM method is faster. Because the four methods use graph structure to represent domain knowledge, there are both similarities and differences among the four methods. Table 2 is the comparison of the four methods, and the comparison shows that the FCPM method has a rapid reason.

Table 2. Comparisons Among Kautz Method, PKG Algorithm, PFA Algorithm and Our Algorithm

\begin{tabular}{|c|l|l|l|l|}
\hline & \multicolumn{1}{|c|}{ Kautz's method } & \multicolumn{1}{|c|}{ PFA algorithm } & \multicolumn{1}{c|}{ PFA algorithm } \\
\hline $\begin{array}{c}\text { Knowledge } \\
\text { structure }\end{array}$ & $\begin{array}{l}\text { Using hierarchical planning } \\
\text { graph to store knowledge. }\end{array}$ & $\begin{array}{l}\text { Using AND/OR graph to store } \\
\text { knowledge, more simple and } \\
\text { clearer. }\end{array}$ & $\begin{array}{l}\text { Similar to PKG,but it } \\
\text { adds the support of } \\
\text { parent node to children. }\end{array}$ & $\begin{array}{l}\text { Similar with PKG, adds support } \\
\text { of parent node to children. }\end{array}$ \\
\hline Search method & $\begin{array}{l}\text { According each observation, } \\
\text { produces a sub-graph. Then, } \\
\text { it checks restriction in } \\
\text { solution graph. }\end{array}$ & $\begin{array}{l}\text { Construct the solution graph } \\
\text { using width first search. }\end{array}$ & $\begin{array}{l}\text { It uses Bottom-up and } \\
\text { Top-down to search } \\
\text { PKG. }\end{array}$ & $\begin{array}{l}\text { Construct solution graph using } \\
\text { bottom up and top-down to } \\
\text { search PKG }\end{array}$ \\
\hline Conflict solution & $\begin{array}{l}\text { Until the final merger, } \\
\text { conflict can be found } .\end{array}$ & $\begin{array}{l}\text { In searching procedure, the } \\
\text { restriction can be checked and } \\
\text { cut the unsatisfied conditions } \\
\text { plan. }\end{array}$ & $\begin{array}{l}\text { It checks restriction on } \\
\text { the observed results in } \\
\text { the first searching. }\end{array}$ & $\begin{array}{l}\text { At first searching.the restriction } \\
\text { is checked. And then it solves the } \\
\text { conflict and predict future } \\
\text { actions. }\end{array}$ \\
\hline Results & $\begin{array}{l}\text { For dealing with the final } \\
\text { result, it proposed a } \\
\text { hypothesis that minimal } \\
\text { planning plan sets were } \\
\text { solution set. }\end{array}$ & $\begin{array}{l}\text { It can not only acquire more } \\
\text { reasonable results, but also get } \\
\text { the occurrence probability of } \\
\text { the plan. }\end{array}$ & $\begin{array}{l}\text { It can get the } \\
\text { occurrence probability } \\
\text { of the plan and predict } \\
\text { future actions. }\end{array}$ & $\begin{array}{l}\text { probability of the plan that is } \\
\text { much more reasonable. } \\
\text { Moreover, it can predict future } \\
\text { actions. }\end{array}$ \\
\hline
\end{tabular}

\section{Conclusions}

It proposes recognizing algorithm based on FCPM. The recognizing algorithm can use matrix operation recognizing plan. Compared with other algorithm, it has excellent performance in plan recognition and the same recognition result is true for PKG algorithm. And it can reduce the recognition time greatly. In order to compare with PKG algorithm easily, it also considers supporting degree of part to whole only, but it doesn't consider the part to whole. So, it can't predict future actions. Adding predicting function is our next work. For example, to use the algorithm based on FCPM in more complex adversarial environment, such as intelligent game, military command, intrusion detection, and so on.

\section{Acknowledgements}

This work is supported by Key Technologies R \& D Program of He'nan Province (172102210059), National Natural Science Foundation of China (No.61672471, and No.61502436) and University Science and Technology Innovation Team of Henan Province.

\section{References}

1. H.B.Barón, R.G.Crespo, J.P.Espada, et al. "Assessment of Learning in Environments Interactive Through Fuzzy Cognitive Maps.” Soft Computing, vol.19, no.4, pp.1037-1050, 2015.

2. M.Bevilacqua , F. E. Ciarapica, and G. Mazzuto. “A Fuzzy Cognitive Maps Tool for Developing a RBI\&M Model.” Quality \& Reliability Engineering International, vol.32, no.2, pp.373-390, 2016.

3. Z. Y. Cai, Y. Feng, Y. Gan, R. Zhang and J. W. Zhang, "Research on Plan Recognition Based on Misleading Action Processing," Open Automation and Control Systems Journal, vol. 6, no. 1, pp. 1029-1037, 2014.

4. Z. Y. Cai, Y. Feng and S. Liu, et al. "A New Intrusion Prevention Model Using Planning Knowledge Graph," International Conference on Graphic and Image Processing (ICGIP 2012), pp. 14-16, Singapore, March 2013.

5. Z. Y Cai, Y. Feng, J. W. Zhang and B. W. Zhang, "Research on Predicting Future Actions Based on Plan Knowledge Graph," 2012 IEEE Symposium on Robotics and Applications, Kuala Lumpur, vol. 6, pp. 961-964, 2012.

6. Z. Y. Cai, Q. K. Zhang, R. Zhang and Y. Gan, "Intrusion Intention Recognition and Response Based on Weighed Plan Knowledge Graph," Computer Modelling and New Technologies, vol. 18, no. 12, pp. 151-157, 2014.

7. E. Charniak and R. P. Goldman, "A Bayesian Model of Plan Recognition," Artificial Intelligence, vol. 64, no. 1, pp. 53-79, 1993.

8. R. P. Goldman and E.Charniak, "Probabilistic Text Understanding," Statistics \& Computing, vol. 2, no. 2, pp. 105-114, 1992. 
9. A. Henry and A. Kautz. "Formal Theory of Plan Recognition," Rochester: University of Rochester, 1987.

10. H. Lee and S. J. Kwon, "Ontological Semantic Inference Based on Cognitive Map," Expert Systems with Applications, vol. 41, no. 6, pp. 2981-2988, 2014.

11. Y. F. Jiang and N. Ma, “A Plan Recognition Algorithm Based on Plan Knowledge Graph”, Journal of Software, vol. 4, pp. 686692, 2002.

12. W. L. Johnson and E. Soloway. "PROUST: Knowledge-Based Program Understanding," IEEE Transactions on Software Engineering, vol.11, no.3, pp. 267-275, 1985.

13. D. Litman and J. Allen, "A Plan Recognition Model for Subdialogues in Conversation," Cognitive Science, vol. 11, no. 1, pp. 163-200, 1987

14. D. McDermott and J. Handler, "Planning: What Is, What It Could be, an Introduction to the Special Issue on Planning and Scheduling," Artificial Intelligence, vol. 76, pp. 1-16, 1995.

15. C. F. Schmidt, N. S. Sridharan and J. L. Goodson, "The Plan Recognition Problem: An Intersection of Psychology and Artificial Intelligence," Artificial Intelligence, vol. 11, no. 1, pp. 45-83, 1978. 